\title{
Étude rétrospective de la croissance en diamètre du sapin baumier (Abies balsamea (L.) Mill.) et de l'épinette rouge (Picea rubens Sarg.) en peuplements mixtes après une coupe à diamètre limite
}

\section{RÉSUMÉ}

La croissance en diamètre à hauteur de poitrine de 341 tiges de sapin baumier et d'épinette rouge a été reconstituée sur un horizon de 50 ans après une coupe à diamètre limite. L'information disponible a été analysée à l'aide d'un modèle statistique dans lequel les effets du temps, de l'essence (deux niveaux), du type écologique (trois niveaux) et du diamètre au moment de la récolte ont été testés. Les résultats révèlent que les gaules de sapin baumier ont des croissances diamétrales supérieures à celles des gaules d'épinette rouge, mais uniquement sur les types écologiques riches. Ces écarts entre les deux espèces s'atténuent chez les tiges dont le diamètre était marchand $(d h p>9,0 \mathrm{~cm})$ au moment de la récolte. Sur le type pauvre, la croissance en diamètre de l'épinette rouge est supérieure à celle du sapin baumier et ce, peu importe le diamètre au moment de la récolte. Par ailleurs, il ressort de l'analyse que le diamètre de la tige au moment de l'intervention est une variable importante dans la prédiction de la croissance en diamètre chez l'épinette rouge, alors que l'effet de cette même variable est beaucoup moins marqué dans le cas du sapin baumier. Bien que significatifs, les écarts de croissance entre les deux espèces sont modestes. La compétition exercée par la régénération de sapin baumier sur les tiges d'épinette rouge n'apparaît donc pas comme un facteur important dans le phénomène de raréfaction de l'épinette rouge.

Mots-clés : épinette rouge (Picea rubens Sarg.), sapin baumier (Abies balsamea (L.) Mill.), croissance en diamètre, coupe partielle, peuplements mixtes, modèle linéaire, modèle mixte

\section{ABSTRACT}

Diameter growth at breast height of 341 balsam fir and red spruce stems has been reproduced over a time lapse of 50 years following a diameter limit cutting. The available information has been analyzed with a statistical model in which the effects of time, species (two levels), ecological type (three levels) and diameter at harvesting have been tested. Results indicate that balsam fir saplings have higher diameter growths than red spruce saplings, but only in the case of better ecological types. The differences between both species are smaller for stems having a merchantable diameter $(d b h>9.0 \mathrm{~cm})$ at harvesting time. On poor ecological station, red spruce diameter growth is more important than for balsam fir and this, whatever the diameter at harvesting time. On the other hand, as indicated by this analysis, stem diameter at the time of silvicultural treatment constitutes a significant variable in the prediction of diameter growth for red spruce stems, while the effect of this variable is less identifiable for balsam fir. Even if significant, the growth differences between the two species are small. The competition created by the balsam fir regeneration on red spruce saplings is definitely not the cause of the depletion of red spruce in second growth stands..

Key words: red spruce (Picea rubens Sarg.), balsam fir (Abies balsamea (L.) Mill.), diameter growth, partial cut, mixed stands, linear model, mixed model

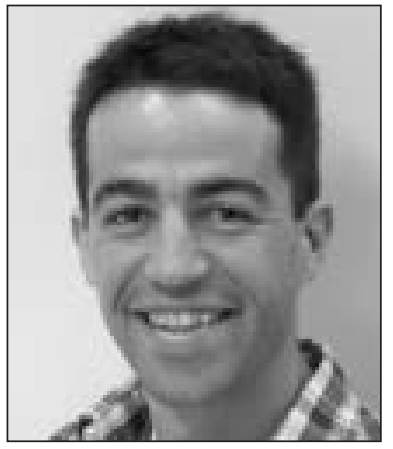

Mathieu Fortin

\section{Introduction}

Les peuplements mixtes de sapin baumier (Abies balsamea (L.) Mill.) et d'épinette rouge (Picea rubens Sarg.) sont typiques de la région forestière acadienne (Rowe 1972). Par conséquent, ils occupent une proportion importante du territoire forestier dans les provinces maritimes du Canada et les états américains du Nord de la Nouvelle-Angleterre.
Au Québec, on retrouve également cette association végétale dans les Basses-Laurentides et les Cantons de l'Est, deux régions qui correspondent au sous-domaine bioclimatique de l'érablière à bouleau jaune de l'Est (Grondin et al. 1996, Gosselin et al. 2001).

Depuis le début du $19^{\text {ème }}$ siècle, ces peuplements ont joué un rôle prépondérant dans l'approvisionnement en matière ligneuse. Offrant un potentiel élevé pour le sciage de fortes dimensions, ils ont d'abord été exploités pour en récolter les tiges de pin blanc et les épinettes de fort diamètre (Ray 1941, Gaudreau 1986, Seymour 1992). À cette époque, les coupes étaient sélectives et l'intensité du prélèvement était légère (Ray 1941, Hatcher 1959). Au tournant du $20^{\text {éme }}$ siècle, l'essor

\footnotetext{
${ }^{1}$ Département des sciences du bois et de la forêt, Faculté de Foresterie et de Géomatique, Université Laval, Sainte-Foy, Québec,

${ }^{2}$ Adresse actuelle : Direction de la recherche forestière, 2700 rue Einstein, Sainte-Foy, Québec G1P 3W8 Tel: 418-643-7994 email: mathieu.fortin@mrnf.gouv.qc.ca
} 
de l'industrie des pâtes et papiers a provoqué une augmentation de la superficie des coupes et de l'intensité du prélèvement (Heimburger 1941, Ray 1956, Seymour 1992).

À partir des années 1940, on s'interroge sur la durabilité de l'aménagement forestier alors pratiqué dans les peuplements mixtes de sapin baumier et d'épinette rouge (e.g. Heimburger 1941). La diminution des proportions d'épinette rouge au profit du sapin baumier après certaines interventions est identifiée comme un problème alarmant, particulièrement sur les stations les plus fertiles (Heimburger 1941, Ray 1941, Hatcher 1959, Seymour 1992, Gordon 1996). Gordon (1996) signale que le taux d'occupation actuel de l'épinette rouge ne représente plus que le cinquième de ce qu'il était dans la forêt précoloniale.

Plusieurs facteurs semblent contribuer à la raréfaction de l'épinette rouge. Bien que cette espèce soit très tolérante à l'ombre et que sa régénération puisse persister pendant plusieurs décennies sous couvert (Blum 1990, Seymour 1992), les semis et les gaules d'épinette rouge sont rarement aussi abondants que ceux de sapin baumier (Ray 1941, Brissette 1996, Fortin et al. 2003a). De plus, les semis d'épinette rouge semblent sensibles aux changements de conditions environnementales et, par le fait même, plus vulnérables que ceux de sapin (Seymour 1992, Alexander et al. 1995). En plus d'être abondante, la régénération du sapin baumier réagit fortement au dégagement (Bakuzis et Hansen 1965, Frank 1990, McCarthy 2001). Après une ouverture du couvert, la régénération de cette espèce pourrait donc induire une forte compétition sur les semis et les gaules d'épinette rouge et ralentir leur croissance (Blum 1990, Seymour 1992).

Cette étude vise à mieux documenter ce problème de compétition, lequel pourrait s'avérer un facteur déterminant dans le phénomène de raréfaction de l'épinette rouge. Pour ce faire, la croissance diamétrale de 341 tiges d'épinette rouge et de sapin baumier a été reconstituée sur un horizon de 50 ans à la suite d'une coupe à diamètre limite. À partir de l'information recueillie, les trois hypothèses suivantes ont été vérifiées à l'aide d'un modèle statistique : (i) à la suite de la coupe, la croissance en diamètre des gaules de sapin baumier est supérieure à celle des gaules d'épinette rouge, (ii) compte tenu que les tiges marchandes d'épinette rouge conservent une capacité de réaction à l'éclaircie jusqu'à des âges avancés (Ray 1956, Blum 1990, Seymour 1992), cette différence de croissance s'atténue chez les tiges marchandes et, enfin, (iii) les écarts de croissance sont plus importants sur les stations les plus fertiles. Les résultats obtenus ont été mis en perspective par rapport aux connaissances actuelles quant à l'autécologie des deux espèces concernées. L'objet de la discussion était de constater dans quelle mesure ce problème de compétition entre les deux espèces contribue au phénomène de raréfaction de l'épinette rouge.

\section{Matériel}

\section{Cadre écologique}

En 1953, le dispositif de l'Aire d'observation de la rivière Ouareau (AORO) $\left(46^{\circ} 26^{\prime} \mathrm{N}, 74^{\circ} 10^{\prime} \mathrm{W}\right)$ a été implanté sur une concession forestière de la Consolidated Paper Corporation. Il est aujourd'hui inclus dans les limites du Parc national du Mont-Tremblant, lequel se situe à environ $100 \mathrm{~km}$ au nord de Montréal. La température annuelle moyenne dans la localité la plus proche, Saint-Donat-de-Montcalm, est de $3,2^{\circ} \mathrm{C}$ pour la période 1971-2000 (Environnement Canada 2002). Juillet est le mois le plus chaud, avec une température moyenne de $18,1^{\circ} \mathrm{C}$, tandis que janvier est le mois le plus froid, avec une valeur moyenne de $-13,6^{\circ} \mathrm{C}$. Les précipitations annuelles moyennes sont de $1128 \mathrm{~mm}$, dont $295 \mathrm{~mm}$ tombent sous forme de neige.

L'AORO a une superficie totale de $13 \mathrm{~km}^{2}$. L'altitude moyenne du dispositif est de $515 \mathrm{~m}$. Les dépôts de surface qui la caractérisent sont majoritairement des tills d'une épaisseur variable. Le relief typique de cette région se compose d'une succession de collines de basse altitude aux sommets arrondis (Robitaille et Saucier 1998).

Le cadre écologique général des Basses-Laurentides a été amplement décrit par plusieurs auteurs (Heimburger 1941, Gagnon et Marcotte 1980, Bélanger 1985, Lalumière et Thibault 1988, Grondin et al. 1996, Gosselin et al. 2001). Les peuplements mixtes de sapin baumier et d'épinette rouge se retrouvent généralement sur les stations à sols minces et sur les stations mésiques situées au bas des pentes et en terrains plats. Selon la classification écologique actuellement utilisée dans la province de Québec, ces stations correspondent aux types écologiques de la bétulaie jaune à sapin (MJ20, MJ22) et de la sapinière à épinette rouge (RS50) (cf. Gosselin et al. 2001). Dans une perspective plus large, la bétulaie jaune à sapin et la sapinière à épinette rouge peuvent être respectivement associées aux types forestiers "Spruce-fir-hardwood " et «Spruce-fir » définis par Westveld (1953).

Aucune donnée d'indice de qualité de station (IQS) n'est disponible pour documenter la richesse des stations sur l'AORO. La structure irrégulière des peuplements mixtes (Hatcher 1954, Fortin et al. 2003a), la tolérance à l'ombre des espèces considérées (Blum 1990, Frank 1990) et l'oppression en bas âge à laquelle elles sont souvent soumises (Ray 1956, Seymour 1992) sont autant de facteurs qui viennent compliquer le calcul de cette variable. Il existe toutefois un lien étroit entre certaines variables écologiques et l'IQS (Bélanger et al. 1995), de sorte qu'une classification écologique peut s'avérer efficace pour discriminer la productivité des stations forestières (Ménard 1999). Sur des types écologiques semblables à ceux de cette étude, Bédard (2002) a démontré que les IQS du sapin baumier et de l'épinette rouge variaient significativement d'un type à l'autre.

La bétulaie jaune à sapin ( "Spruce-fir-hardwood») possède une richesse relative plus élevée que la sapinière à épinette rouge ("Spruce-fir ») (Heimburger 1941, Gosselin 2001). La richesse relative se définit ici comme la synthèse de plusieurs variables écologiques, notamment la présence de drainage oblique, le $\mathrm{pH}$ de l'humus, le type d'humus et la diversité floristique (cf. Gosselin et al. 2001). Pour alléger le texte, les références ultérieures aux types écologiques ont été faites en fonction de leur richesse relative, la bétulaie jaune à sapin étant le type riche et la sapinière à épinette rouge étant le type pauvre.

Le type riche est caractérisé par des peuplements mélangés dont les essences principales sont le sapin baumier, l'épinette rouge, le bouleau jaune (Betula alleghaniensis Britton) et le bouleau à papier (Betula papyrifera Marsh.) (Heimburger 1941, Grondin et al. 1996, Gosselin et al. 2001). Les essences qui les accompagnent sont l'érable à sucre (Acer saccharum Marsh.), l'érable rouge (Acer rubrum L.) et le thuya (Thuja occidentalis L.). Les peuplements qui croissent sur le type pau- 
vre se caractérisent par une dominance des essences résineuses, soit l'épinette rouge et le sapin baumier (Heimburger 1941, Grondin et al. 1996, Gosselin et al. 2001). Le bouleau à papier et le thuya y sont présents, mais de façon sporadique.

\section{Historique des perturbations}

Depuis 1938, deux épidémies de tordeuse des bourgeons de l'épinette (Choristoneura fumiferana Clem.) ont eu lieu dans ce secteur, la première durant les décennies 1940-50 et la seconde durant celles de 1970-80 (Hardy et al. 1987). Il n'y a pas eu d'infestation sévère à l'intérieur du dispositif lors de la première épidémie (Hatcher 1954). Par ailleurs, les travaux d'inventaires à l'été 1998 et l'été 1999 n'ont pas permis d'identifier des zones de mortalité sévère, ce qui suggère que les effets de la seconde épidémie ont été limités en termes de mortalité. Des carottes de croissance prélevées sur des tiges de sapin baumier et d'épinette rouge ont permis d'obtenir une estimation de l'accroissement annuel courant (AAC) en diamètre à hauteur de poitrine $(d h p$, mesuré à $1,30 \mathrm{~m})$ pour la période 1954-98 (Fig. 1a, 1b). Chez les deux espèces, les effets de la seconde épidémie se sont traduits par une diminution d'accroissement pour une période d'environ cinq ans au milieu des années 1970. On observe également une diminution d'accroissement chez le sapin baumier pour une deuxième période de cinq ans durant les années 1980. La diminution des années 1980 est moins marquée que celle de la décennie précédente. De manière générale, les défoliations n’ont pas été critiques au point d'inhiber la croissance. Au pire de l'épidémie, les deux espèces ont en effet maintenu des AAC supérieurs ou équivalents à $1 \mathrm{~mm}$ par année.

En ce qui a trait à l'exploitation forestière, une récolte de bois à pâte a été réalisée durant les saisons hivernales entre l'automne 1947 et le printemps 1956. Le traitement alors en vigueur était la récolte des résineux dont le diamètre à la souche était équivalent ou supérieur à $18 \mathrm{~cm}$ (7 po). Les tiges ont été abattues à la sciotte et au godendart, tandis que le débardage s'est effectué à cheval et à traîneau (Hatcher 1954). Entre 50 et $60 \%$ du volume total des tiges de plus de $8,9 \mathrm{~cm}$ $(3,5$ po) au $d h p$ ont été récoltés lors de cette intervention (Hatcher 1954).

\section{Dispositif de suivi}

Le dispositif de suivi de l'AORO a été établi en deux blocs à l'été 1953. Au total, il compte 288 placettes permanentes réparties systématiquement dans chacun des blocs selon une grille dont les maillons sont espacés de $201 \mathrm{~m}$. Chaque placette a une superficie de $405 \mathrm{~m}^{2}$ (0,1 acre). À l'intérieur de chacune d'elles, toutes les tiges dont le $d h p$ était supérieur à $1,3 \mathrm{~cm}(0,5$ po) ont été dénombrées par classe de $2,5 \mathrm{~cm}$ (1 po). Le dispositif a été mesuré à nouveau en 1963 et entre 1974 et 1977. En 1998 et 1999, 59 placettes ont été mesurées pour une quatrième fois.

\section{Récolte et compilation des données}

Au printemps 1999, 13 placettes du dispositif ont fait l'objet d'une étude détaillée de la croissance en diamètre. Ces placettes ont été choisies parmi celles (i) coupées lors de l'intervention de 1947-56, (ii) retrouvées lors de l'inventaire de 1998-99 et (iii) correspondant à l'un ou l'autre des deux types écologiques retenus. À l'intérieur de ces 13 placettes, toutes les

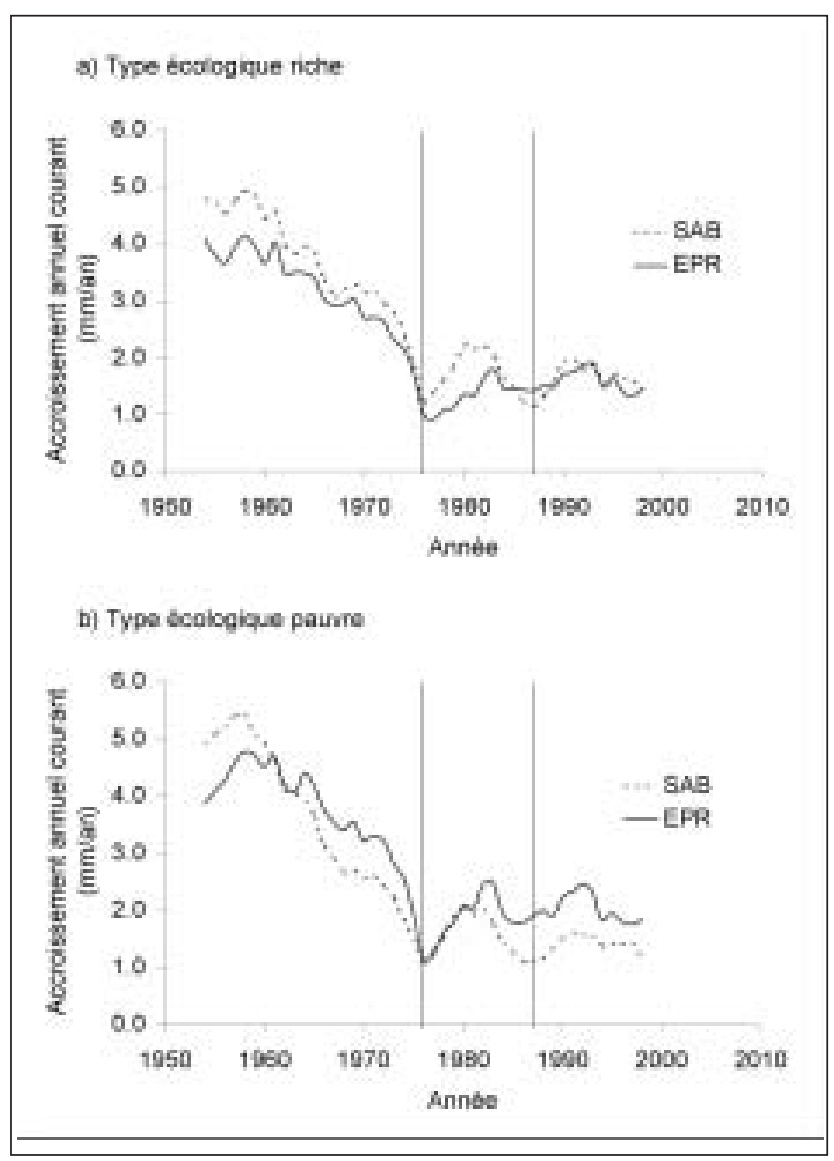

Fig. 1. Accroissements annuels courants (AAC) en diamètre à hauteur de poitrine $(d h p)$ des tiges de sapin baumier (SAB) et d'épinette rouge (EPR) entre 1954 et 1998 sur l'Aire d'observation de la rivière Ouareau.

tiges de sapin baumier et d'épinette rouge dont le $d h p$ était supérieur ou équivalent à 63 mm (2,5 po) en 1999 ont été échantillonnées pour en connaître le diamètre, l'épaisseur de l'écorce et la croissance en diamètre depuis la coupe de 1947-56. Le diamètre a été mesuré au millimètre près à l'aide d'un ruban diamétrique. Trois mesures de l'épaisseur de l'écorce ont été effectuées à hauteur de poitrine à l'aide d'une jauge à écorce. Ces mesures ont été prises au millimètre près selon un angle de 120 degrés l'une par rapport à l'autre. Trois carottes ont ensuite été prélevées à hauteur de poitrine à la sonde de Pressler selon une configuration semblable à celle des mesures d'écorce, c'est-à-dire à un angle de 120 degrés l'une par rapport à l'autre. Au total, 465 tiges ont été échantillonnées.

En 2000, tous les échantillons ont été séchés et sablés pour ensuite en mesurer la largeur des cernes annuels à l'aide du logiciel WinDendro. Trois années repères ont été utilisées pour dater les échantillons. Cette mesure visait à éviter l'omission de cernes manquants ou peu apparents en raison des défoliations résultant des épidémies de tordeuse. Pour estimer la largeur des cernes à l'état vert, des retraits de 2,7\% et 4,0\% ont été respectivement utilisés pour le sapin baumier et l'épinette rouge (Jessome 1977).

À l'été 2003, les 13 placettes étudiées ont été visitées et classifiées à nouveau en raison de précisions apportées au système 
Tableau 1. Compositions et densités des trois types écologiques en 1953 (les valeurs entre parenthèses indiquent l'écart type)

\begin{tabular}{|c|c|c|c|}
\hline Caractéristiques & $\begin{array}{l}\text { Type riche } \\
\text { très mince }\end{array}$ & $\begin{array}{l}\text { Type riche } \\
\text { épais }\end{array}$ & $\begin{array}{l}\text { Type pauvre } \\
\text { très mince }\end{array}$ \\
\hline Nbre de placettes & 4 & 6 & 3 \\
\hline \multicolumn{4}{|l|}{ Bouleau jaune } \\
\hline Surface terrière $\left(\mathrm{m}^{2} \cdot \mathrm{ha}^{-1}\right)$ & $0,3(0,5)$ & $2,2(1,7)$ & $0,0(0,0)$ \\
\hline Densité (ti·ha-1) & $30(29)$ & $41(33)$ & $9(15)$ \\
\hline \multicolumn{4}{|l|}{ Bouleau à papier } \\
\hline Surface terrière $\left(\mathrm{m}^{2} \cdot \mathrm{ha}^{-1}\right)$ & $2,8(1,0)$ & $1,4(1,3)$ & $0,2(0,2)$ \\
\hline Densité $\left(\mathrm{ti} \cdot \mathrm{ha}^{-1}\right)$ & $868(339)$ & $261(210)$ & $193(80)$ \\
\hline \multicolumn{4}{|l|}{ Épinette rouge } \\
\hline Surface terrière $\left(\mathrm{m}^{2} \cdot \mathrm{ha}^{-1}\right)$ & $7,6(3,5)$ & $4,0(2,8)$ & $5,3(2,5)$ \\
\hline Densité $\left(\mathrm{ti}^{\mathrm{i}} \mathrm{ha}^{-1}\right)$ & $1044(265)$ & $427(290)$ & $764(239)$ \\
\hline \multicolumn{4}{|l|}{ Sapin baumier } \\
\hline Surface terrière $\left(\mathrm{m}^{2} \cdot \mathrm{ha}^{-1}\right)$ & $9,5(7,9)$ & $8,3(3,6)$ & $2,7(1,5)$ \\
\hline Densité $\left(\right.$ ti $\left.\cdot h^{-1}\right)$ & $3213(2608)$ & $1878(585)$ & $837(249)$ \\
\hline \multicolumn{4}{|l|}{ Autres essences } \\
\hline Surface terrière $\left(\mathrm{m}^{2} \cdot \mathrm{ha}^{-1}\right)$ & $0,8(1,6)$ & $2,3(5,5)$ & $0,0(0,0)$ \\
\hline Densité $\left(\right.$ ti $\left.\cdot \mathrm{ha}^{-1}\right)$ & $75(151)$ & $12(30)$ & $0(0)$ \\
\hline \multicolumn{4}{|l|}{ Toutes essences confondues } \\
\hline Surface terrière $\left(\mathrm{m}^{2} \cdot \mathrm{ha}^{-1}\right)$ & $21,1(8,6)$ & $18,1(7,1)$ & $8,3(4,1)$ \\
\hline Densité $\left(\mathrm{ti} \cdot \mathrm{ha}^{-1}\right)$ & $5230(3040)$ & $2618(914)$ & $1802(465)$ \\
\hline
\end{tabular}

Tableau 2. Sommaire des données utilisées pour la calibration du modèle (les valeurs entre parenthèses indiquent l'écart type)

\begin{tabular}{lccc}
\hline Caractéristiques & $\begin{array}{c}\text { Type riche } \\
\text { très mince }\end{array}$ & $\begin{array}{c}\text { Type riche } \\
\text { épais }\end{array}$ & $\begin{array}{c}\text { Type pauvre } \\
\text { très mince }\end{array}$ \\
\hline $\begin{array}{l}\text { Épinette rouge } \\
\text { Nbre de tiges } \\
\text { dhpinit }(\mathrm{mm})\end{array}$ & 50 & 44 & 40 \\
$\begin{array}{l}\text { Sapin baumier } \\
\text { Nbre de tiges } \\
\text { dhpinit }\end{array}$ & $59(104)$ & $48(102)$ & $46(81)$ \\
\hline
\end{tabular}

adhpinit : dhp avec écorce au moment de la coupe

de classification écologique provincial (cf. Gosselin 2001). Cette nouvelle classification a permis d'obtenir trois groupes homogènes à partir des 13 placettes initialement retenues : le type riche sur dépôt très mince (4 placettes), le type riche sur dépôt épais (6 placettes) et le type pauvre sur dépôt très mince (3 placettes). Le seuil de $25 \mathrm{~cm}$ est utilisé dans cette classification pour qualifier l'épaisseur du dépôt (Gosselin 2001). Le tableau 1 présente un portrait sommaire des placettes en fonction des trois types écologiques retenus. Ce sommaire a été compilé à partir des données de l'inventaire de 1953, soit l'inventaire réalisé dans les premières années suivant la coupe.

Afin de retracer l'évolution du diamètre avec écorce (dhpae) à partir des carottes de croissance, les mesures d'épaisseur d'écorce ont d'abord été utilisées pour établir une relation entre le diamètre avec écorce et le diamètre sans écorce ( $d h p s e)$. Les équations obtenues par régression linéaire sont présentées en annexe. Les accroissements annuels en dhpse ont ensuite été calculés en prenant la moyenne arithmétique de la largeur des cernes d'une année donnée pour les carottes d'une même tige et en multipliant cette moyenne par deux. L’année 1998 a été considérée comme la dernière année de croissance complète. Le dhpse 1997 a été estimé en soustrayant l'accroissement annuel de $1998 \mathrm{du}$ dhpse ${ }_{1998}$. Cette démarche a été répétée autant de fois que nécessaire pour obtenir tous les dhpse entre l'année de la coupe et 1998. Les dhpae correspondant à chacune de ces années ont été calculés en utilisant la relation préalablement établie entre le dhpae et le dhpse.

Les tiges pour lesquelles il était impossible d'estimer le dhpae au moment de la coupe ont été retirées de l'analyse. Parmi les 124 arbres rejetés, 80 tiges (21 épinettes et 59 sapins) étaient des recrues, c'est-à-dire des tiges qui n'avaient pas de $d h p$ au moment de la coupe. Pour les 44 autres tiges 
(5 épinettes et 39 sapins), les carottes étaient cariées ou trop endommagées pour prendre des mesures. Au total, 341 tiges ont été retenues pour l'analyse, chacune d'elles comptant en moyenne 48 à 49 mesures. Le tableau 2 présente la répartition de ces tiges selon les différents types écologiques.

\section{Méthode}

Les données ont d'abord été soumises à un examen préliminaire afin de faciliter l'élaboration d'un modèle. À partir du patron général d'accroissement, le modèle suivant a été retenu :

$$
\text { [1] } \Delta \text { dhpae }=\beta_{1} \ln (t+1)+\beta_{2} \ln ^{2}(t+1)
$$

où $\Delta$ dhpae représente la croissance en $d h p$ avec écorce après la coupe $(\mathrm{mm}), t$, le nombre d'années écoulées depuis la récolte et $\beta_{1}$ et $\beta_{2}$, les paramètres du modèle. Le modèle [1] est linéaire, polynomial du second degré et ne comporte pas d'ordonnée à l'origine. Pour tester les hypothèses de départ, chacun des deux paramètres du modèle $\left(\beta_{1}\right.$ et $\left.\beta_{2}\right)$ a été remplacé par une fonction linéaire exprimant trois effets, soit l'essence, le type écologique et le diamètre au moment de la coupe (dhpinit) ainsi que les interactions possibles entre ceux-ci.

Au point de vue statistique, les données servant à l'analyse posent certains problèmes. D'une part, les placettes constituent un blocage dans le plan d'expérience, ce qui représente un effet aléatoire. D'autre part, les mesures d'accroissement sont des mesures répétées. Le fait d'avoir échantillonné le même arbre plus d'une fois laisse supposer que les erreurs issues de ces mesures sont corrélées entre elles. Les analyses qui font abstraction de cette corrélation fournissent des estimés non biaisés des paramètres (Sullivan et Clutter 1972, Vanclay 1994). Par contre, elles biaisent les variances qui sont associées à ces estimés, affectant ainsi le choix des variables explicatives à inclure dans le modèle (Gregoire et al. 1995).

Les effets aléatoires et les effets des mesures répétées peuvent être pris en compte en estimant la structure de la matrice de variance-covariance des erreurs (Littell et al. 1996). Pour les effets aléatoires, on ajoute à la variance du modèle une composante pour tenir compte de chaque effet aléatoire (Gregoire 1987, Littell et al. 1996). Cette composante de la variance permet de contrôler des effets qui ne sont pas inclus dans le modèle sous forme de variables explicatives (Gregoire 1987). Les effets aléatoires peuvent être associés à l'ordonnée à l'origine ou à certaines variables indépendantes sous forme d'interaction (Gregoire et al. 1995, Littell et al. 1996). En ce qui concerne l'effet des mesures répétées, plusieurs structures de covariance permettent de définir la relation qui existe entre ces mesures. Parmi les plus connues, la structure autorégressive définit une corrélation qui diminue en fonction de la distance entre les mesures. À l'inverse, la structure symétrique ( « compound symmetry ») conserve une corrélation constante, peu importe le nombre d'unités de temps séparant les mesures répétées.

Dans le cas présent, deux composantes ont été ajoutées à la variance du modèle, chacune correspondant à un effet aléatoire. Ces effets ont été inclus dans chacune des deux fonctions remplaçant les deux paramètres du modèle [1] et ont pris la forme d'une interaction entre l'essence et la placette. Cette interaction apparaissait logique, les effets incontrôlables dus à la placette pouvant être différents d'une espèce à l'autre. En ce qui concerne les mesures répétées, une matrice de variance-covariance non structurée a d'abord été utilisée pour identifier la structure de covariance la plus appropriée, conformément à la méthode décrite dans Littell et al. (1996). Toutes les analyses ont été effectuées à l'aide de la procédure MIXED du progiciel SAS (SAS Institute 2002).

\section{Résultats}

Une calibration préliminaire du modèle a permis de détecter une structure de covariance autorégressive de premier ordre dans la matrice de variance-covariance des erreurs. Les résidus ont également affiché une hétéroscédasticité marquée. La structure autorégressive à variance hétérogène (cf. Littell et al. 1996) a été retenue pour les essais subséquents. Toutefois, l'utilisation de cette structure de covariance implique l'estimation d'une variance propre à chaque intervalle de temps en plus d'un paramètre établissant la corrélation entre les intervalles successifs. Dans le cas présent, cette structure supposait l'estimation de 52 paramètres. Pour alléger le processus, seuls des intervalles de cinq ans ont été conservés. Le nombre de paramètres est ainsi passé de 52 à 11 .

Le modèle a été calibré de nouveau en incluant cette nouvelle structure de variance-covariance et en ne conservant que les effets et interactions significatifs à un seuil $\alpha=5 \%$. Les résidus standardisés de cette nouvelle calibration affichaient les caractéristiques requises pour assurer la validité des inférences statistiques, soit une variance homogène, une distribution normale et des corrélations très faibles. Le tableau 3 présente les résultats de la calibration.

\section{Tableau 3. Résultats de la calibration du modèle}

\begin{tabular}{lc}
\hline Éléments de l'analyse & Valeur \\
\hline Vraisemblance (-2Loglikelihood) & 19962 \\
Nombre d'observations & 3191 \\
Corrélation entre deux mesures successives & 0,9879 \\
Erreur type $(\mathrm{mm})$ & \\
10 ans après la coupe & 20,9 \\
30 ans après la coupe & 33,9 \\
50 ans après la coupe & 43,5 \\
Effets aléatoires $($ variance $)$ & \\
Essence $\times$ Placette $\times \ln (t+1)$ & 6,56 \\
Essence $\times$ Placette $\times \ln 2(t+1)$ & 1,96 \\
Effets fixes $($ Pr $>$ F) & \\
$\ln ^{2}(t+1)$ & $<0,0001$ \\
Essence $\times \ln (t+1)$ & 0,0470 \\
Type écologique $\times$ Essence $\times \ln (t+1)$ & 0,0115 \\
dhpinit $\times \ln (t+1)$ & 0,0118 \\
dhpinit $\times$ Essence $\times \ln (t+1)$ & $<0,0001$ \\
dhpinit $\times$ Type écologique $\times \ln (t+1)$ & 0,0007 \\
$\ln ^{2}(t+1)$ & $<0,0001$ \\
Essence $\times \ln ^{2}(t+1)$ & 0,0099 \\
Type écologique $\times$ Essence $\times \ln { }^{2}(t+1)$ & 0,0477 \\
dhpinit $^{\mathrm{a}} \times \ln ^{2}(t+1)$ & $<0,0001$ \\
dhpinit $\times$ Essence $\times \ln { }^{2}(t+1)$ & $<0,0001$ \\
\hline
\end{tabular}

${ }^{a}$ dhpinit : dhp avec écorce au moment de la coupe 

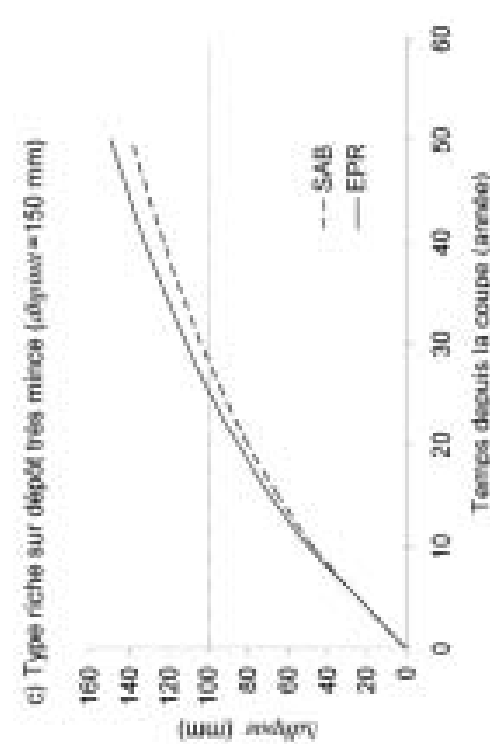

(uni) molum

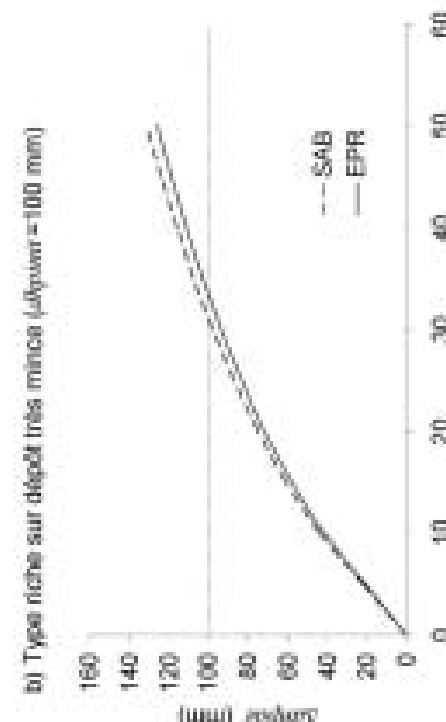

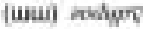

8

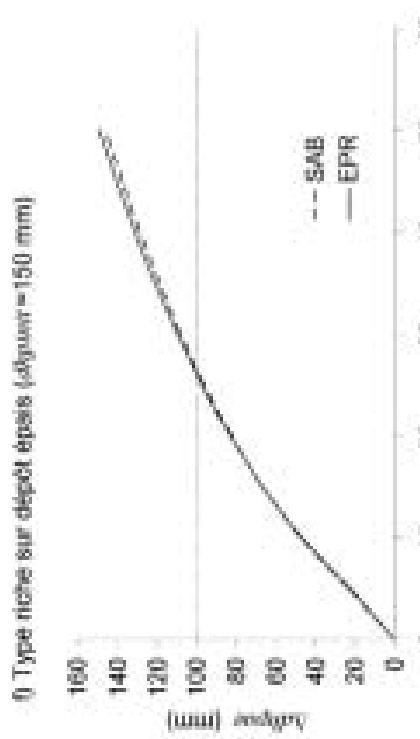

웅

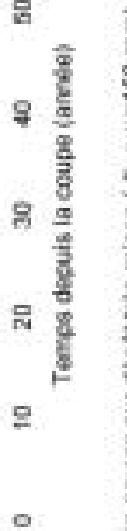

(umi) malyy

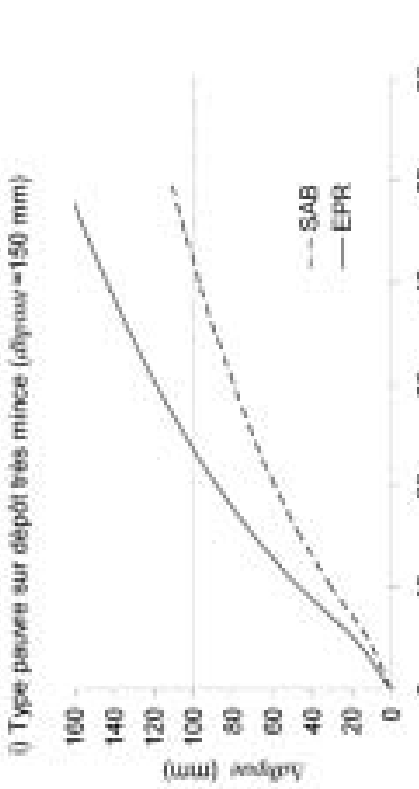

뭄

몸

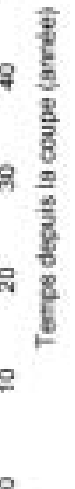

웅

(uai) molyos

8

i) 密皆

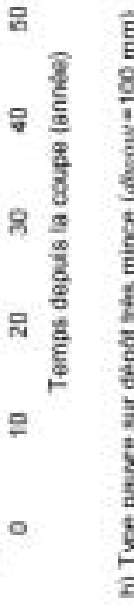

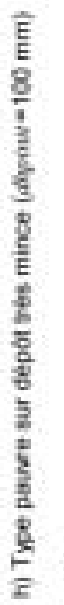

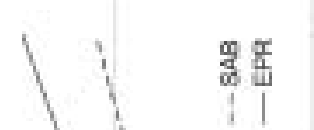

뭄

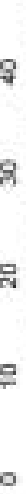

8 으옹요융ㅇㅇㅇ

(uiu) noffors

욨

8

(uii) nodye

요
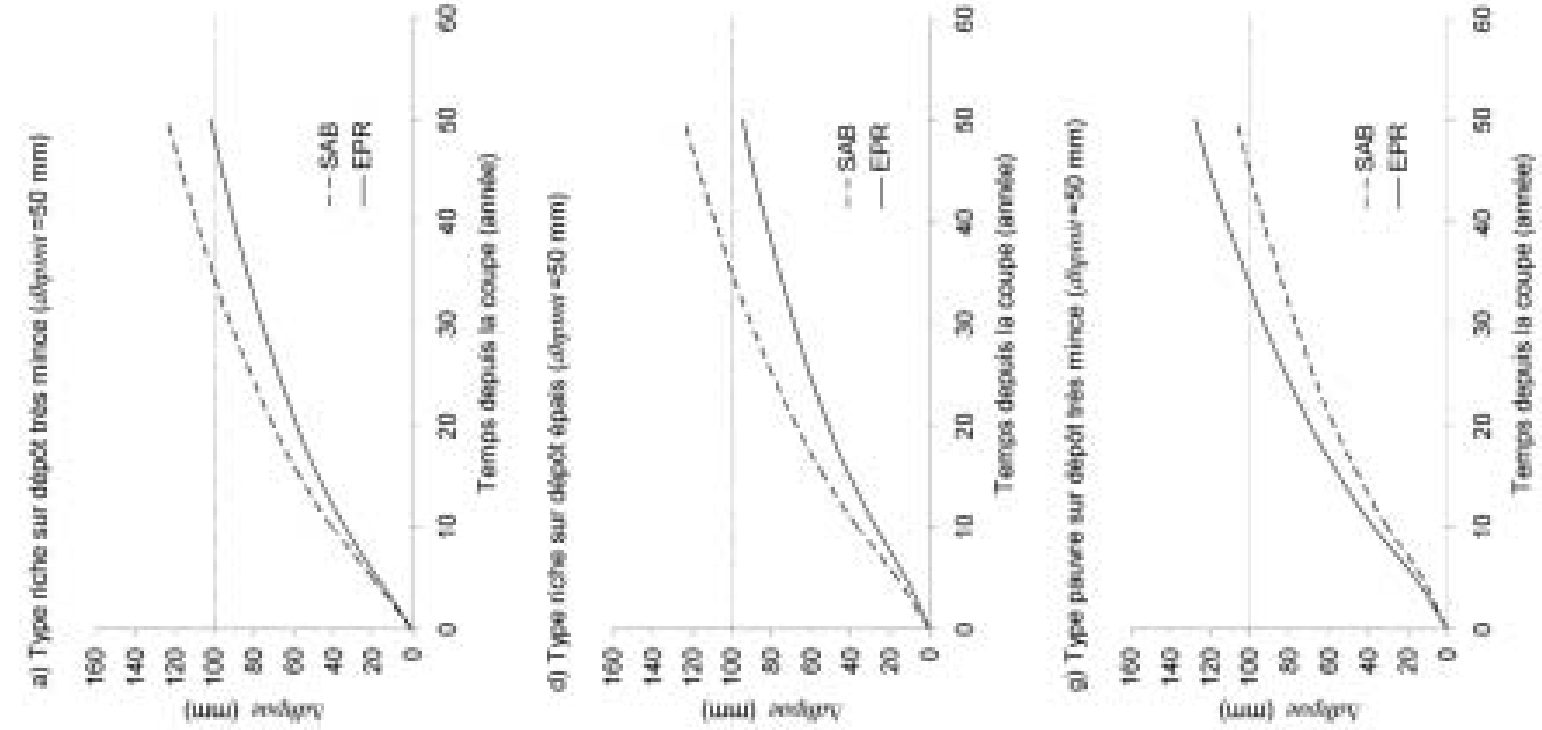

(uiu) montoy 
La corrélation entre deux mesures successives de cinq ans est très élevée et atteint près de $99 \%$. L'erreur type augmente avec le temps, démontrant l'hétéroscédasticité des données. L'erreur passe ainsi d'une valeur de $20,9 \mathrm{~mm}, 10$ ans après coupe, à une valeur de $43,5 \mathrm{~mm}, 50$ ans après coupe. Les effets aléatoires dus aux placettes ont des variances respectives de 6,56 et 1,96 .

Les probabilités associées aux effets fixes démontrent que la croissance diamétrale du sapin baumier est significativement différente de celle de l'épinette rouge, l'essence étant présente dans le modèle sous forme d'interaction avec le temps (Essence $\times \ln (t+1)$ et Essence $\times \ln ^{2}(t+1)$ ). De plus, cette différence de croissance entre les deux essences varie en fonction du type écologique (Type écologique $X$ Essence $X$ $\ln (t+1)$ et Type écologique $\times$ Essence $\left.\times \ln ^{2}(t+1)\right)$.

Le diamètre des tiges au moment de l'intervention (dhpinit) a également un effet significatif sur la croissance diamétrale à la suite de l'intervention, cette variable explicative étant, elle aussi, présente sous forme d'interaction avec le temps (dhpinit $\times \ln (t+1)$ et dhpinit $\times \ln ^{2}(t+1)$ ). Cette interaction entre le diamètre initial et le temps varie significativement d'une espèce à l'autre (dhpinit $\times$ Essence $\times \ln (t+$ 1 ) et dhpinit $\times$ Essence $\left.\times \ln ^{2}(t+1)\right)$ et d'un type écologique à l'autre $($ dhpinit $\times$ Type écologique $\times \ln (t+1))$. Les croissances diamétrales prédites par le modèle [1] sont présentées à la Fig. 2 en fonction de l'essence, du type écologique et de trois diamètres initiaux (dhpinit). Les équations à partir desquelles sont tirées ces courbes sont présentées en annexe. Les croissances atteignent entre 95 et $165 \mathrm{~mm}$ sur un horizon de 50 ans, ce qui correspond à des accroissements annuels moyens (AAM) variant entre 1,9 et 3,3 mm-an ${ }^{-1}$. Les patrons de croissance des types riches sont relativement semblables, alors que celui du type pauvre differe.

Sur les types riches, les tiges de sapin baumier d'un diamètre initial (dhpinit) de $50 \mathrm{~mm}$ affichent une croissance moyenne plus rapide que celles d'épinette rouge (Fig. 2a, 2d). Chez les deux espèces, l'augmentation du diamètre initial se traduit par une augmentation de la croissance. Cette augmentation est toutefois plus importante chez l'épinette rouge, de sorte que la croissance diamétrale de cette espèce en vient à rattraper et même à dépasser légèrement celle du sapin baumier chez les tiges dont le diamètre initial est de $150 \mathrm{~mm}$ (Fig. 2c, 2f).

Sur le type pauvre, l'épinette rouge affiche une croissance moyenne supérieure à celle du sapin baumier et ce, pour chacun des trois exemples présentés (Fig. 2g, 2h, 2i). Quant à l'augmentation du diamètre initial, elle se traduit par une augmentation de la croissance, mais seulement dans le cas de l'épinette rouge. En effet, les courbes du sapin baumier sur le type pauvre sont pratiquement identiques dans les trois cas.

Pour un même diamètre initial, la croissance en diamètre des tiges d'épinette rouge sur le type pauvre est plus rapide que celle sur les types riches. Chez le sapin baumier, la tendance inverse est observée : la croissance moyenne des types riches est plus rapide que celle du type pauvre.

\section{Discussion}

Le sapin baumier est une espèce opportuniste qui profite à la fois des petites et des grandes trouées pour réagir fortement à la mise en lumière (Bakuzis et Hansen 1965, Frank 1990, McCarthy 2001). De façon générale, les gaules d'épinette rouge sont moins nombreuses, plus susceptibles d'être affectées par les changements de conditions environnementales et leur réaction à l'éclaircie n'est pas aussi forte (Ray 1956, Frank et Bjorkbom 1973, Seymour 1992). On présume donc que les gaules d'épinette souffriraient de la compétition exercée par le sapin baumier (Blum 1990, Seymour 1992).

À la suite d'une coupe à diamètre limite, les gaules de sapin baumier ont effectivement une croissance diamétrale moyenne supérieure à celle des gaules d'épinette rouge sur les types écologiques riches. Bien que significatives, les différences sont toutefois modestes. Sur un horizon de 10 ans, soit durant les premières années suivant la récolte, la différence entre les deux espèces est en moyenne inférieure à 10 $\mathrm{mm}$ pour des tiges dont le $d h p$ au moment de la coupe était de $50 \mathrm{~mm}$ (Fig. 2a, 2d). Les résultats de la présente étude portent donc à conclure que la compétition exercée par le sapin baumier sur les types écologiques riches après une coupe à diamètre limite a une incidence limitée sur la croissance diamétrale des gaules d'épinette rouge. Non seulement les écarts de croissance entre les deux espèces sont relativement faibles, mais les erreurs types du modèle sont beaucoup plus élevées que ces écarts (tableau 3). Si l'on sélectionnait une gaule de chaque espèce au hasard, il ne serait donc pas improbable que la gaule d'épinette rouge ait une croissance plus rapide que celle de sapin baumier, bien que la tendance générale favorise le sapin.

Par ailleurs, les différences de croissance observées entre les gaules des deux espèces s'atténuent lorsque le diamètre initial des tiges augmente. Il en ressort que le diamètre de la tige au moment de l'intervention est un facteur d'importance dans la prédiction de la croissance en diamètre chez l'épinette rouge, alors que l'effet de cette même variable est beaucoup moins marqué dans le cas du sapin baumier. Bien que le patron de croissance soit différent sur le type pauvre, on remarque ce même effet " diamètre » chez l'épinette rouge.

La capacité de réaction du sapin baumier à la suite d'une éclaircie tend à diminuer, puis à se stabiliser, en fonction de la hauteur initiale de la tige (Hatcher 1964, Riopel 1999). Un dégagement favorise donc les tiges de sapin de faible diamètre (Bakuzis et Hansen 1965). Après une coupe partielle, Riopel (1999) estime que les tiges de $6 \mathrm{~m}$ de hauteur sont celles qui affichent un accroissement maximum. Alors que la longévité du sapin baumier n'excède pas 200 ans (Bakuzis et Hansen 1965, Frank 1990), l'épinette rouge peut atteindre des âges supérieurs à 400 ans (Ray 1956, Blum 1990, Busing et Wu 1990), ce qui en fait l'une des espèces les plus longévives du Québec. Même lorsqu'elle atteint un âge très avancé, l'épinette rouge conserve une étonnante capacité de réaction à l'éclaircie (Robertson 1942, Ray 1956, Seymour 1995). En effet, même les arbres très âgés peuvent s'accroître significativement, du moment que leurs cimes ont l'espace nécessaire pour s'étendre (Seymour et Kenefic 2002). Par ailleurs, même si les tiges d'épinette rouge sont souvent soumises à des périodes d'oppression (Blum 1990, Seymour 1992), la croissance à la suite du dégagement s'en trouve peu affectée (Seymour et Fajvan 2001). En somme, l'effet « diamètre » qui s'observe chez l'épinette rouge s'explique par le fait que cette espèce peut maintenir une efficacité de croissance relativement bonne en dépit de l'âge et de l'historique du peuplement.

Alors que l'on attendait, dans une certaine mesure, un patron semblable à celui des types riches, la croissance diamé- 
trale des gaules de sapin baumier est en moyenne inférieure à celle des gaules d'épinette rouge sur le type pauvre. Toutefois, la différence de croissance entre les deux espèces est peu importante, soit de l'ordre de $20 \mathrm{~mm}$ sur un horizon de 50 ans (Fig. 2g) pour des tiges dont le $d h p$ était de $50 \mathrm{~mm}$ au moment de l'intervention. À cause de l'effet du diamètre initial chez l'épinette rouge, cet écart de croissance tend à augmenter en fonction du diamètre des tiges au moment de la récolte.

Si le patron de croissance des gaules sur le type pauvre est surprenant d'un point de vue écologique, il est néanmoins conforme à ce que l'on connait de la productivité des types écologiques étudiées. Sur des stations comparables à celles de cette étude, Bédard (2002) a en effet démontré que l'IQS de l'épinette rouge augmente légèrement sur le type pauvre, passant de $16,2 \mathrm{~m}$ à $17,2 \mathrm{~m}$ en prenant 50 ans comme âge de référence. À l'inverse, l'IQS du sapin baumier tend à diminuer fortement, passant de 19,2 $\mathrm{m}$ sur les types riches à une valeur de 16,4 $\mathrm{m}$ sur le type pauvre pour le même âge de référence (Bédard 2002). En outre, à des densités équivalentes, la croissance en volume de l'épinette rouge à l'échelle du peuplement ne varie pas de façon significative d'un type écologique à l'autre, alors que celle du sapin baumier est beaucoup plus faible sur le type écologique pauvre (Fortin et al. 2003b). Les résultats de la présente étude vont donc dans le même sens que ceux des études de Bédard (2002) et Fortin et al. (2003b) : par rapport aux types écologiques riches, la croissance diamétrale de l'épinette rouge connaît une légère augmentation sur le type pauvre, alors que celle du sapin baumier diminue de façon significative.

L'ajout d'une mesure de densité parmi les effets fixes du modèle aurait permis d'améliorer la précision de l'analyse de la croissance en diamètre des deux espèces. Plusieurs essais de modélisation ont été réalisés en utilisant les surfaces terrières et les nombres de tiges à l'hectare calculés à partir de l'inventaire de 1953. Dans tous les cas, ces variables se sont avérées non significatives. En théorie, les effets aléatoires prennent compte des variables de site qui ne sont pas incluses comme effets fixes dans un modèle mixte (Gregoire 1987). Toutefois, dans le cas présent, les densités sont associées aux types écologiques (tableau 1). En effet, une analyse de variance (ANOVA) réalisée sur les surfaces terrières des types écologiques indique un lien étroit entre les deux variables $(\operatorname{Pr}=0,0348)$, les surfaces terrières les plus faibles se retrouvant essentiellement sur le type pauvre. Les deux variables étant fortement associées, la capacité de la régression à ne pas les confondre peut raisonnablement être mise en doute. L'effet du type écologique reflète donc une interaction indissociable entre cette variable et la densité. Un plan d'expérience offrant une meilleure dispersion des densités initiales permettrait de mieux distinguer ces deux effets l'un de l'autre. En l'absence de telles données, les résultats obtenus par rapport aux types écologiques doivent être interprétés avec prudence, même s'ils semblent conformes à ceux obtenus par d'autres auteurs dans des contextes semblables (e.g. Bédard 2002, Fortin et al. 2003b).

Par ailleurs, cette étude n'aborde que la croissance des tiges qui ont survécu sur un horizon de près de 50 ans. L'effet des deux épidémies de tordeuse sur la croissance de ces tiges est plutôt faible et ne représente, tout au plus, qu'un retard de quelques années. Les tiges mortes durant cet intervalle sont probablement celles qui affichaient les accroissements les plus faibles et qui ont été les plus touchées par la tordeuse. Dans le cadre de cette étude, aucune donnée ne permettait d'évaluer la croissance de ces tiges. Cette lacune incite à croire qu'une utilisation des équations issues du modèle [1] à des fins de prédiction sur un horizon inférieur à 50 ans pourrait mener à surestimer la croissance des tiges des deux espèces concernées.

\section{Conclusions}

Sur l'Aire d'observation de la rivière Ouareau, les proportions d'épinette rouge ont fortement diminué sur le type écologique de la bétulaie jaune à sapin après une coupe à diamètre limite (Fortin et al. 2003a). Cette raréfaction de l'épinette rouge ne semble pas être le résultat d'une compétition exercée par les gaules de sapin baumier. Dans les faits, les écarts de croissance en diamètre entre les deux espèces sont en moyenne relativement faibles. Sur le type écologique de la sapinière à épinette rouge, certains auteurs ont signalé que le problème de raréfaction de l'épinette rouge n'était pas alarmant (Ray 1956, Hatcher 1959, Fortin et al. 2003b). Les résultats de cette étude vont dans le même sens en démontrant que, sur ce même type écologique, les gaules d'épinette rouge réagissent plus fortement à l'éclaircie que celles de sapin baumier.

L'abondance de la régénération de chacune des deux espèces est sans doute un facteur qui contribue plus fortement aux changements de composition que la croissance ellemême. Après une coupe partielle, la régénération du sapin baumier est généralement plus abondante que celle de l'épinette rouge (Heimburger 1941, Ray 1956, Brissette 1996, Archambault et al. 2003). Comme la régénération de l'épinette rouge demeure relativement constante dans le temps, la raréfaction de l'espèce semble plus le résultat d'un prélèvement trop important que d'une régénération déficiente (Fortin et al. 2003a). Or, on connait peu les facteurs qui régissent l'établissement de la régénération d'épinette rouge. Aussi, cette avenue de recherche pourrait se révéler un domaine clé dans la sylviculture de cette espèce.

\section{Remerciements}

L'auteur tient à remercier le Fonds forestier du ministère des Ressources naturelles du Québec pour le financement de ce projet, le Service canadien des forêts pour le prêt des données de l'Aire d'observation de la rivière Ouareau, le Parc national du Mont-Tremblant pour l'accès au dispositif, le personnel de terrain ayant participé à la récolte et au traitement des échantillons, le Conseil de recherches en sciences naturelles et en génie du Canada (CRSNG) et la compagnie AbitibiConsolidated inc. (bureau Grand-Mère) pour leur soutien financier. Je tiens également à remercier le professeur Jean Bégin (Université Laval), M. Robert Schneider (Université Laval), Mme Marie-Josée Verreault (Université Laval) ainsi que quatre examinateurs anonymes pour leurs commentaires quant à une version préliminaire de ce manuscrit. En dernier lieu, il importe de souligner le travail exceptionnel de M. Robert J. Hatcher, qui a supervisé l'établissement et le suivi du dispositif de l'Aire d'observation de la rivière Ouareau. 


\section{Références}

Alexander, J.D., J.R. Donnelly et J.B. Shane. 1995. Photosynthetic and transpirational responses of red spruce understory trees to light and temperature. Tree Physiol. 15: 393-398.

Archambault, L., J. Bégin, C. Delisle et M. Fortin. 2003. Succession forestière après coupe partielle dans la Forêt expérimentale du Lac Édouard, Parc de la Mauricie, Québec. For. Chron. 79(3): 672-684.

Bakuzis, E.V. et H.L. Hansen. 1965. Balsam fir. A monographic review. Univ. of. Minnesota Press. Minneapolis. 445 p.

Bédard, S. 2002. L'estimation du potentiel de croissance des stations forestières : exemple du sous-domaine de la sapinière à bouleau jaune de l'est du Québec. Ministère des Ressources naturelles du Québec, Direction de la recherche forestière. Mémoire de recherche forestière no. $140.36 \mathrm{p}$.

Bélanger, L. 1985. Intégration de l'information écologique dans la gestion des terres de l'écoumène forestier par l'inventaire écologique des terres. Thèse de doctorat. Université Laval. 599 p.

Bélanger, L., S. Paquette, S. Morel, J. Bégin, P. Meek, L. Bertrand, P. Beauchesne, S. Lemay et M. Pineau. 1995. Indices de qualité de station du sapin baumier dans le sous-domaine écologique de la sapinière à bouleau blanc humide. For. Chron. 71(3): 317-325.

Blum, B.M. 1990. Red Spruce. Dans Silvics of North America, Vol. 1. pp. 250-259. USDA Agriculture Handbook no 654.

Brissette, J.C. 1996. Effects of intensity and frequency of harvesting on abundance, stocking and composition of natural regeneration in the Acadian forest of Eastern North America. Silva Fennica 30(2-3): 301-314.

Busing, R.T. et X. Wu. 1990. Size specific mortality, growth, and structure of a Great Smoky Mountains red spruce population. Can. J. For. Res. 20: 206-210.

Environnement Canada 2002. Normales et moyennes climatiques au Canada 1971-2000 [en ligne]. La Voie verte. Environnement Canada. Disponible à http://climate.weatheroffice.ec.gc.ca/ climate_normals/index_f.html [mis à jour le 10 mai 2004, cité le 27 juillet 2004].

Fortin, M., J. Bégin et L. Bélanger. 2003a. Évolution de la structure diamétrale et de la composition des peuplements mixtes de sapin baumier et d'épinette rouge de la forêt primitive après une coupe à diamètre limite sur l'Aire d'observation de la rivière Ouareau. Can. J. For. Res. 33: 691-704.

Fortin, M., J. Bégin et L. Bélanger. 2003b. Les coupes partielles : une alternative à la coupe à blanc dans les peuplements mixtes de sapin baumier et d'épinette rouge en termes de rendement. For. Chron. 79(5): 948-956.

Frank, R.M. 1990. Balsam fir. Dans Silvics of North America, Vol. 1. pp. 26-35. USDA Agriculture Handbook no 654.

Frank, R.M. et J.C. Bjorkbom. 1973. A silvicultural guide for spruce-fir in the Northeast. USDA For. Serv. Gen. Tech. Rep. NE-6. 29 p.

Gagnon, G. et G. Marcotte. 1980. Description des types écologiques et de leur productivité dans la section forestière laurentienne de Rowe (L-4a). Gouvernement du Québec. Ministère de l'Énergie et des Ressources. Service de la recherche forestière. Mémoire no. 61. $456 \mathrm{p}$.

Gaudreau, G. 1986. L'exploitation des forêts publiques au Québec 1842-1905. Institut québécois de recherche sur la culture. Québec. $126 \mathrm{p}$.

Gordon, A.G. 1996. The sweep of the boreal in time and space, from forest formations to genes, and implications for management. For. Chron. 72(1) : 19-30.

Gosselin, J. 2001. Guide de reconnaissance des types écologiques de la région écologique $3 c-$ Hautes collines du Bas-Saint-Maurice. Forêt Québec. Direction des inventaires forestiers.

Gosselin, J., P. Grondin et J.-P. Saucier. 2001. Rapport de classification écologique du sous-domaine bioclimatique de l'érablière à bouleau jaune de l'est. Ministère des Ressources naturelles du Québec, Direction de la gestion des stocks forestiers. 169 p.
Gregoire, T.G. 1987. Generalized error structure for forestry yield models. For. Sci. 33(2): 423-444.

Gregoire, T.G., O. Schabenberger et J.P. Barrett. 1995. Linear modelling of irregularly spaced, unbalanced, longitudinal data from permanent-plot measurements. Can. J. For. Res. 25: 137-156.

Grondin, P., C. Ansseau, L. Bélanger, J.-F. Bergeron, Y. Bergeron, A. Bouchard, J. Brisson, L. De Grandpré, G. Gagnon, C. Lavoie, G. Lessard, S. Payette, P.J.H. Richard, J.-P. Saucier, L. Sirois et L. Vasseur. 1996. Écologie forestière. Dans Manuel de foresterie. pp. 133-279. Les Presses de l'Université Laval, Sainte-Foy.

Hardy, Y., M. Mainville et D.M. Schmitt. 1987. An atlas of spruce budworm defoliation in Eastern North America, 1938-80. USDA For. Serv. Misc. Pub. No. 1449. 52 p.

Hatcher, R.J. 1954. A report on the establishment of observation area no. 12 on the limits of the Consolidated Paper Corporation Ouareau River, P.Q. 1953. Government of Canada. Department of Northern Affairs and National Resources. Forestry Branch. Forest Research Division. Project Q-54.31 p.

Hatcher, R.J. 1959. Partial cutting with diameter limit control in the Lake Edward Experimental Forest, Quebec, 1950 to 1956. Government of Canada. Department of Northern Affairs and National Resources. Forestry Branch. Forest Research Division. Project Q-44. 8 p.

Hatcher, R.J. 1964. Balsam fir advance growth after cutting in Québec. For. Chron. 40(1): 86-92.

Heimburger, C.C. 1941. Forest site classification and soil investigation in the Lake Edward Forest Experimental Area. Government of Canada. Department of Mines and Resources. Lands, Parks and Forest Branch. Dominion Forest Service. Silvicultural Research Note No. 66.60 p.

Jessome, A. P. 1977. Résistance et propriétés connexes des bois indigènes au Canada. Forintek Canada Corp. Publication SP-514F. Sainte-Foy. 37 p.

Lalumière, R. et M. Thibault. 1988. Les forêts du Parc national de la Mauricie au Québec. Étude écologique no 11. Presses de l'Université Laval, Sainte-Foy. 495 p.

Littell, R.C., G.A. Milliken, W.W. Stroup et R.D. Wolfinger. 1996. SAS System for mixed models. SAS Institute Inc., Cary, NC. 633 p.

McCarthy, J. 2001. Gap dynamics of forest trees: A review with particular attention to boreal forests. Environ. Rev. 9: 1-59.

Ménard, B. 1999. Dynamique naturelle des forêts mixtes de la station expérimentale du lac Édouard, au Parc national de la Mauricie. Mémoire de maîtrise. Université Laval. 87 p.

Ray, R.G. 1941. Site-types and rate of growth. Government of Canada. Department of Mines and Resources. Lands, Parks and Forest Branch. Dominion Forest Service. Silvicultural Research Note No. 65.63 p.

Ray, R.G. 1956. Site-types, growth and yield at Lake Edward Experimental Area Quebec. Government of Canada. Department of Northern Affairs and National Resources. Forestry Branch. Forest Research Division. Technical Note No. 27. 53 p.

Riopel, M. 1999. Potentiel de croissance de la haute régénération préétablie de sapin baumier : une analyse dendrométrique au niveau de la tige et du peuplement. Mémoire de maîtrise. Université Laval. $149 \mathrm{p}$.

Robertson, W.M. 1942. Some growth characteristics of red spruce. Government of Canada. Department of Mines and Resources. Lands, Parks and Forest Branch. Dominion Forest Service. Silvicultural Research Note No. 70.7 p.

Robitaille, A. et J.-P. Saucier. 1998. Paysages régionaux du Québec méridional. Ministère des Ressources naturelles du Québec. 213 p.

Rowe, J.S. 1972. Les régions forestières du Canada. Ministère de l'environnement du Canada. Service canadien des forêts. Publication no 1300F. 172 p.

SAS Institute. 2002. SAS OnlineDoc 9 [en ligne]. SAS Institute inc. Cary, NC. Disponible à http://v9doc.sas.com/sasdoc/. 
Seymour, R.S. 1992. The red spruce-balsam fir forest of Maine: Evolution of silvicultural practice in response to stand development patterns and disturbances. Dans M.J. Kelty, B.C. Larson et C.D. Oliver (eds.). The ecology and silviculture of mixed-species forests. pp. 217-244. Kluwer Publishers, Norwell, MA.

Seymour, R.S. 1995. The northeast region. Dans J.W. Barrett (ed.). Regional silviculture of the United States, $3^{\text {rd }}$ Ed. pp. 31-79.

Seymour, R.S. et M.A. Fajvan. 2001. Influence of prior growth suppression and soil on red spruce site index. North. J.. Appl. For. 18(2): $55-62$.

Seymour, R.S. et L.S. Kenefic. 2002. Influence of age on growth efficiency of Tsuga canadensis and Picea rubens trees in mixed-species, multiaged northern conifer stands. Can. J. For. Res. 32: 2032-2042.

Sullivan, A.D. et J.L. Clutter. 1972. A simultaneous growth and yield model for loblolly pine. For. Sci. 18: 76-86.

Vanclay, J.K. 1994. Modelling forest growth and yield, applications to mixed tropical forests. $\mathrm{CAB}$ International. $312 \mathrm{p}$.

Westveld, M. 1953. Ecology and silviculture of the spruce-fir forests of eastern North America. J. For. 51: 422-430.

\section{Annexes}

\section{Relations dhpae vs dhpse}

Pour le sapin baumier,

$$
\text { dhpse }=9,50 \times 10^{-1} \cdot \text { dhpae }+3,71 \times 10^{-5} \cdot \text { dhpae }^{2}
$$

Pour l'épinette rouge,

$$
\text { dhpse }=9,41 \times 10^{-1} \cdot \text { dhpae }+5,68 \times 10^{-5} \cdot \text { dhpae }^{2}
$$

où dhpae représente le diamètre à hauteur de poitrine avec écorce $(\mathrm{mm})$ et $d h p s e$, le diamètre à hauteur de poitrine sans écorce $(\mathrm{mm})$.

\section{Équations tirées du modèle [1]}

Type écologique riche sur dépôt très mince

Pour le sapin baumier,

$$
\begin{aligned}
& \Delta \text { dhpae }=\left(-6,49+8,87 \times 10^{-3} \text { dhpinit } 1 \mathrm{n}(t+1)+\right. \\
& \left(9,15+7,47 \times 10^{-3} \text { dhpinit }\right) 1 \mathrm{n}^{2}(t+1),
\end{aligned}
$$

Pour l'épinette rouge,

$$
\begin{gathered}
\Delta \text { dhpae }=\left(-2,93-5,18 \times 10^{-2} \text { dhpinit } \ln (t+1)+\right. \\
\left(5,80+3,74 \times 10^{-2} \text { dhpinit }\right) 1 \mathrm{n}^{2}(t+1)
\end{gathered}
$$

Type écologique riche sur dépôt épais

Pour le sapin baumier,

$$
\begin{aligned}
& \Delta \text { dhpae }=\left(-1,14 \times 10^{1}+2,67 \times 10^{-2} \text { dhpinit }\right) 1 \mathrm{n}^{2}(t+1) \\
& \quad+\left(1,02 \times 10^{1}+7,47 \times 10^{-3} \text { dhpinit }\right) 1 \mathrm{n}^{2}(t+1)
\end{aligned}
$$

Pour l'épinette rouge,

$$
\begin{aligned}
& \Delta \text { dhpae }=\left(-7,81-8,39 \times 10^{-3} \text { dhpinit }\right) \ln (t+1) \\
& +\left(6,33+3,74 \times 10^{-2} \text { dhpinit }\right) 1 \mathrm{n}^{2}(t+1)
\end{aligned}
$$

Type écologique pauvre sur dépôt très mince

Pour le sapin baumier,

$$
\begin{aligned}
& \Delta \text { dhpae }=\left(-9,32-1,67 \times 10^{-2} \text { dhpinit }\right) \ln (t+1) \\
& +\left(9,09+7,47 \times 10^{-3} \text { dhpinit }\right) 1 \mathrm{n}^{2}(t+1)
\end{aligned}
$$

Pour l'épinette rouge,

$$
\begin{aligned}
& \Delta \text { dhpae }=\left(-8,05-5,18 \times 10^{-2} \text { dhpinit }\right) \ln (t+1) \\
& +\left(9,07+3,74 \times 10^{-2} \text { dhpinit }\right) 1 \mathrm{n}^{2}(t+1)
\end{aligned}
$$

où $\Delta$ dhpae représente la croissance en diamètre à hauteur de poitrine avec écorce après la coupe $(\mathrm{mm}), t$ représente le nombre d'années écoulées depuis la coupe et dhpinit, le diamètre au moment de la récolte $(\mathrm{mm})$. 